Research Paper

\title{
Effect of Administration of Lidocaine at Body Temperature on Anesthesia Success in Rodent Model: A Behavioral and Electrophysiology Study
}

\author{
Sina Kakooei ${ }^{1,2}$ (D), Mohammadreza Afarinesh ${ }^{2,3,4^{*}}$ (D), Masoud Parirokh ${ }^{5}$ (D), Reza Nikzad ${ }^{6}$ (D), Mahshid Mostafavi ${ }^{7}$ (D), Amir Nekouei (D) $^{-}$ \\ Mansoureh Sabzalizadeh ${ }^{3}$ (D), Vahid Sheibani ${ }^{3,7}$ (D) \\ 1. Oral and Dental Diseases Research Center, Dental School, Kerman University of Medical Sciences, Kerman, Iran \\ 2. Neuroscience Research Center, Institute of Neuropharmacology, Kerman University of Medical Sciences, Kerman, Iran. \\ 3. Student Research Committee, School of Medicine, Kerman University of Medical Sciences, Kerman, Iran. \\ 4. Cognitive Neuroscience Research Center, Institute of Neuropharmacology, Kerman University of Medical Sciences, Kerman, Iran. \\ 5. Endodontology Research Center, Dental School, Kerman University of Medical Sciences, Kerman, Iran. \\ 6. Department of Clinical Sciences, Faculty of Veterinary Medicine, Shahid Bahonar University of Kerman, Kerman, Iran. \\ 7. Leishmaniasis Research Center, Kerman University of Medical Sciences, Kerman, Iran.
}

\begin{tabular}{|c|c|}
\hline $\begin{array}{l}\text { Use your device to scan } \\
\text { and read the article online }\end{array}$ & dtation Kakooei, S., Afarinesh, M. R., Parirokh, M., Nikzad, R., Mostafavi, M., \& Nekouei, A., et al. (2022). Effect \\
\hline 口itis: & $\begin{array}{l}\text { of Administration of Lidocaine at Body Temperature on Anesthesia Success in Rodent Model: A Behavioral and Electro- } \\
\text { physiology Study. Basic and Clinical Neuroscience, 13(3), 305-314. http://dx.doi.org/10.32598/bcn.2022.1102.2 }\end{array}$ \\
\hline 口sters & dol'http://dx.doi.org/10.32598/ben.2022.1102.2 \\
\hline
\end{tabular}

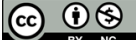

Article info:

Received: 26 Jan 2021

First Revision: 25 Jul 2021

Accepted: 11 Oct 2021

Available Online: 01 May 2022

Keywords:

Lidocaine, Local anesthesia,

Formalin test, Rat, Success rate

\begin{abstract}
AB S T RAC T
Introduction: Success in anesthesia administration relieves the perception of pain during surgery. Lidocaine is the most commonly used local anesthetic agent in clinical medicine. Moreover, anesthetic agents' temperature changes can influence cell membrane permeability. Here, the effectiveness of different temperatures of Lidocaine (Lid.) on anesthesia success rate has been investigated in rats.

Methods: Wistar male rats were pretreated by fast injection of lidocaine or saline into the hind paw or intradermal cheek at Room Temperature (RT) and Body Temperature (BT) $\left(22^{\circ} \mathrm{C}\right.$ and $37^{\circ} \mathrm{C}$, respectively). Then, rat behaviors were evaluated by formalin-induced hind paw pain and orofacial pain tests, respectively. Moreover, using a single-unit recording technique, the spontaneous activity of the marginal nerve was recorded at room temperature in the RT-Lid. and BT-Lid. groups.

Results: Data analysis revealed that lidocaine had significant antinociceptive effects in both the BT-Lid. and RT-Lid. groups compared to the control groups $(\mathrm{P}<0.05)$. Also, the number of spikes in the BT-Lid. and RT-Lid. groups were significantly lower than their baselines $(\mathrm{P}<0.05)$. However, lidocaine at body temperature decreased the total time spent licking the hind paw, the number of lip rubbings, and the number of spikes firing by about $10 \%-15 \%$ compared to room temperature.

Conclusion: In both behavioral and neural levels of the study, our results showed that an increase in the temperature of lidocaine toward body temperature could increase anesthesia success rate compared to administration of lidocaine at room temperature. These findings can be considered in the treatment of patients.
\end{abstract}

* Corresponding Author:

Mohammad Reza Afarinesh, PhD.

Address: Kerman Neuroscience Research Center, Kerman University of Medical Sciences, Kerman, Iran.

Tel: +98 (34) 32232872

E-mail: rafarinesh@kmu.ac.ir315; reza.afarinesh@gmail.com 


\section{Highlights}

- Lidocaine at body temperature acted better than room temperature on pain control in the formalin-induced hind paw test.

- Lidocaine at body temperature acted better than room temperature on pain control in the orofacial formalin test.

- Lidocaine with different temperatures decreased the firing rate of the marginal nerve.

\section{Plain Language Summary}

Pain is defined as an unpleasant experience caused by tissue damage or fear of injury. During anesthetic injection in dentistry, pain has long been one of the problems of dentists. Studies have shown that one out of every three people is worried about going to dentistry, and one of four dental patients is afraid of injections. The fear of a patient in one of twenty patients is so much that interferes with dental treatment which consequently leads to stress when you visit the dentist, results in less oral hygiene and reduces the number of referrals. Lidocaine is the most commonly used local anesthetic agent in clinical medicine. Here, the effectiveness of different temperatures of lidocaine on anesthesia success rate in rats has been investigated in rats. The present study showed that warming the lidocaine cartridges to $37^{\circ} \mathrm{C}$ increased anesthesia success compared to anesthesia-induced at room temperature in both behavioral and neural levels of the study. Accordingly, a warmed anesthetic cartridge could be used to control pain by increasing the success rate during dental injection and designed a new animal model study for further investigation in comparing other anesthesia drugs.

\section{Introduction}

rofacial pain sensation is related to in-

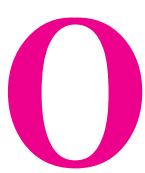
traoral and extraoral structures of the head and face, which are innervated by trigeminal ganglion neurons (Lazarov, 2002). The orofacial formalin test, which involves the injection of formalin into the lips of rats, is a behavioral model for studying the physiology and modulation of trigeminal nociception (Luccarini, Childeric, A., Gaydier, Voisin, \& Dallel, 2006). On the other hand, trigeminal and facial branches are both mixed cranial nerves that carry sensory and motor information about the face, including those of the whiskers, maxilla, mandible, and teeth (Hogan, vanderVaart, Perampaladas, Machado, Einarson, \& Taddio, 2014). In particular, the ventral buccal nerve and marginal mandibular branches of the facial and mandibular branches of the trigeminal nerves exhibit a complicated branching pattern and form communications with each other (Cobo, Solé-Magdalena, Menéndez, de Vicente, \& Vega, 2017; Dörfl, 1985; Hwang, Yang, \& Song, 2015; König, Hans-Georg, \& Bragulla, 2007). It has been shown that neurons in rat trigeminal subnucleus caudalis, a nucleus in the medulla, receive information about dental pain (Carstens, Kuenzler, N., \& Handwerker, 1998), touch, and temperature from the ipsilateral face (Carstens et al., 1998; Klein, Carstens, \& Carstens, 2011).
Pain is an unpleasant sensation that can arise from inflammatory, surgical, or anesthetic injections. Pharmacological and non-pharmacological methods are used to reduce pain (Arendt \& Tessmer-Tuck, 2013; Pahlavan et al., 2013; Yazdkhasti \& Pirak, 2016). Various anesthetic solutions, such as lidocaine, an amide anesthetic, are available to relieve the perception of clinical pain (Rosivack, Koenigsberg, \& Maxwell, 1990; Vickers \& Punnia-Moorthy, 1992). The efficacy, safety, and pharmacokinetics of drugs have been studied in different animal models to improve the quality of the anesthetic blocking properties (McGowan Jr \& Davis, 2008). The solubility of anesthetic agents may increase with increasing temperature (Courtney, Agrawal, \& Revington, 1999; Powell, 1987). Patients who receive local anesthesia report that warmed lidocaine significantly reduces pain (Courtney et al., 1999; Hogan et al., 2011; Lundbom et al., 2017). Moreover, warm-up (Ganji, Shirvani, Rezaei-Abhari, \& Danesh, 2013) and cool-down (Dörfl, $1985)$ of the painful area can reduce pain because sensory inputs to the spinal cord can inhibit the crossing of pain signals by "closing the gate" (Adams \& Bianchi, 2008). However, none of the previous studies (Courtney et al., 1999; Hogan et al., 2011; Lundbom et al., 2017) has examined the effects of temperature on pain relief, particularly in the face area. The effect of lidocaine temperature on the reduction of visceral pain has been considered. Although visceral and physical pains have a similar mechanism for pain detection, the type of pain sensation a person experiences, the pain receptors, the 
pain transmission pathway, and the type of pain response are different. Therefore, the study of pain due to formalin injection in the face and hind legs is generally different from the mentioned studies (Cervero, 2009). By using the formalin test, present study investigates the effects of Lidocaine (Lid.) temperature on acute and chronic pain in the hind paw under controlled conditions of body temperature and room temperature.

On the other hand, in electrophysiology studies, a reduction in neuronal firing is considered representative of a reduction in pain sensation (Carstens et al., 1998; Klein et al., 2011). The single-unit recording technique is an invasive approach that investigates the activity of neurons more applicable to humans. The effects of lidocaine temperature changes on the orofacial formalin test and neural activity of the ventral buccal nerve as models for studying the success rate of anesthesia in rats were also evaluated. This study may benefit clinicians, particularly dentists, because the results show that better pain control may be achieved during injection and surgery by increasing the temperature of the local anesthetic.

\section{Materials and Methods}

\section{Study animals}

Male Wistar rats weighing $250 \pm 25 \mathrm{~g}$ were used in this experiment. The animals were kept in standard housing conditions with a 12:12 h light/dark cycle. Food and water were provided ad libitum. All experimental procedures were performed under protocols approved by the Ethics Committee of the Research Council of Kerman University of Medical Sciences (IR.KMU. REC.1398.679) and the Institutional Animal Care and Use Committee.

\section{Behavioral study}

\section{Formalin-induced hind paw test}

The formalin-induced hind paw test was performed in a plexiglass chamber $(25 \times 25 \times 25 \mathrm{~cm})$ with a controlled floor temperature of $22^{\circ} \mathrm{C}$ (room temperature [RT]) or $37^{\circ} \mathrm{C}$ (Body Temperature [BT]). A mirror was placed underneath at a $45^{\circ}$ angle to allow an unimpeded view of the rats. The rats were randomly divided into five groups $(\mathrm{n}=7)$. Lidocaine $(2 \%, 0.1 \mathrm{~mL})$ (Perzocaine, DaruPakhsh, and Tehran, Iran) (Inan et al., 2009) was injected into their right hind legs to block sensation on the dorsal side of the hind paw. The control groups received Saline (Sal) in the same volume. In the RT-Sal and RT-Lid. groups, subjects received saline or lidocaine at room temperature $\left(22^{\circ} \mathrm{C}\right)$. In the BT-Sal and BT-Lid. groups, subjects received the lidocaine at $37^{\circ} \mathrm{C}$ temperature. Also, during the experiment in these groups, the plexiglass floor temperature was fixed at $37^{\circ} \mathrm{C}$ using a heater. Saline or lidocaine was injected $10 \mathrm{~min}$ before intraplantar injection of formalin ( $50 \mu \mathrm{L}$ of $5 \% \mathrm{v} / \mathrm{v})$ into the right hind-paw (Dubuisson \& Dennis, 1977). The control rats only experienced an intraplantar injection of formalin. The animal was immediately returned to the plexiglass chamber. Animals were observed from the start to $5 \mathrm{~min}$ (acute phase) and then from 15 to $60 \mathrm{~min}$ (chronic phase). The total time spent licking and biting the injected paw was recorded and considered indicative of nociception (Dubuisson \& Dennis, 1977).

\section{Orofacial formalin test}

The orofacial formalin test was performed according to a previous study (Patel et al., 2013). Rats were randomly divided into five groups, as mentioned above $(\mathrm{n}=7)$. In the BT-Sal, RT-Sal, BT-Lid., and RT-Lid. groups, the subjects were injected in the lower right lip with saline $(0.1 \mathrm{~mL})$ or $2 \%$ lidocaine $(0.1 \mathrm{~mL})$ (Perzocaine, DaruPakhsh, and Tehran, Iran) (Inan et al., 2009) using a 30 -gauge needle fitted to a $50 \mu \mathrm{L}$ Hamilton syringe. Ten minutes later, the animals received a subcutaneous injection of formalin $(25 \mu \mathrm{L}, 2 \% \mathrm{v} / \mathrm{v})$ in the lower right lip (Patel et al., 2013). The control rats only received a subcutaneous injection of formalin. Each rat was placed in a clear plastic box $(25 \times 25 \times 25 \mathrm{~cm})$ with a mirror underneath at a $45^{\circ}$ angle to create an unimpeded view of the rats. The number of rubbings of the lip was recorded from 0 to $5 \mathrm{~min}$ (acute phase) and then from 15 to $60 \mathrm{~min}$ (chronic phase). The results were considered indicators of nociception (Patel et al., 2013).

\section{Electrophysiology study}

In the electrophysiological study, 21 animals were randomly divided into two groups. Here, in the RT group $(\mathrm{n}=10)$, lidocaine $2 \%$ was injected at room temperature with a volume of $0.07 \mathrm{~mL}$, while in the BT group $(\mathrm{n}=11)$, the temperature of lidocaine was $37^{\circ} \mathrm{C}$. An insulin syringe plunger (Dena, Karachi, Pakistan) and 27-gauge short needles (Sofijet, Mazamet, France) were used for intradermal injection after nerve exposure.

\section{Electrophysiology procedure}

\section{Recording}

The rats were anesthetized by an intraperitoneal injection of urethane anesthetic $(1.2 \mathrm{~g} / \mathrm{kg})$, and then the 
animals were fixed by the stereotactic frame in the supine position. The ventral buccal (marginal) nerve was exposed after making an incision in the mandibular skin (Heaton et al., 2014). For the single-unit recording technique, a single metal electrode was inserted into the marginal nerve by a micro driver (WPI, USA). An E-Lab device (Parto Danesh, Iran) was utilized to record the activity of neurons. Then $10 \mathrm{~min}$ of the spontaneous activity of the marginal nerve was recorded, ensuring that the base activity of the neuron remained stable. Then the anesthetic solution was injected by the intradermal cheek method (the injection area was in the animal's rostrum, 1 $\mathrm{cm}$ from the exposed nerve). The activities of the single neurons were recorded for $60 \mathrm{~min}$ (Figure 3). A homoeothermic blanket control unit (Harvard, Holliston, USA) was used to reduce heat exchange; it was set to $37^{\circ} \mathrm{C}$ by putting the heat sensor inside the animal's anus. At the end of the procedure, the animals were sacrificed via deep anesthesia with $\mathrm{CO}_{2}$ (Herfst \& Brecht, 2008; Hohman, Kleiss, Knox, Weinberg, Heaton, \& Hadlock, 2014).

\section{Statistical analysis}

Using a post-stimulus time histogram (PSTH), the neural activities of the marginal nerve were recorded at intervals of 0-3000 s (Figure 3). Then, the number of spikes per equal bin (bin=250 s) was calculated as the response of neurons. After the Kolmogorov-Smirnov normality test, one-way ANOVA and two-way ANOVA followed by Bonferroni post hoc tests were used to compare the total time spent licking, the total number of rubbing the lip, and the number of spikes per bin. The data were presented as Mean \pm SEM, and $\mathrm{P}<0.05$ was considered statistically significant.

\section{Results}

Effects of lidocaine at different temperatures on formalin-induced hind paw pain

The one-way ANOVA test followed by the Bonferroni test showed a statistically significant difference in the total time spent licking the hind paw among the treatment groups in both acute $\left(\mathrm{F}_{4,30}=183.3, \mathrm{P}<0.001\right)$ and chronic $\left(\mathrm{F}_{4,30}=210.3, \mathrm{P}<0.001\right)$ phases. ANOVA results showed no statistically significant difference among the control, RT-Sal, and BT-sal groups in acute or chronic phases.

Figure 1 shows that total time spent licking the hind paw decreased significantly in both RT-Lid. and BT-Lid. groups compared to control groups $(\mathrm{P}<0.001)$ during acute and chronic phases. Moreover, in the acute phase, lidocaine had significant antinociceptive effects in the RT-Lid. group (81.6\%) and the BT-Lid. group (98.7\%) compared to the control groups $(\mathrm{P}<0.05)$. Also, in the chronic phase, lidocaine had significant antinociceptive effects in the RT-Lid. group (72.4\%) and the BT-Lid. group $(93.2 \%)$ compared to the control groups $(\mathrm{P}<0.05)$

Effects of lidocaine at different temperatures on formalin-induced orofacial pain

One-way ANOVA followed by the Bonferroni test showed a significant difference in the number of rubbings among treatment groups in the acute $\left(\mathrm{F}_{4,30}=122.0\right.$, $\mathrm{P}<0.001)$ and chronic $\left(\mathrm{F}_{4,30}=84.8, \mathrm{P}<0.001\right)$ phases. At room or body temperature, the saline groups did not indicate any statistical significance in the number of rubbings compared to the control group in both acute and chronic phases. Further analyses showed that the BT-Lid. and RT-Lid. groups had a significant reduction in the number of lip rubbings as compared to the control groups $(\mathrm{P}<0.001)$ during acute and chronic phases of the orofacial pain model (Figure 2). However, there is no significant statistical difference between RT-Lid. and BT-Lid. groups, but lidocaine decreased the number of rubbings in the RT-Lid. (88.2\%) and the BT-Lid. (97.3\%) groups compared to the control group in the acute phase. In the chronic phase, lidocaine at room and body temperatures had an antinociceptive effect on the RT-Lid. (83.8\%) and BT-Lid. (91.7\%) groups compared to the control groups, respectively.

Effects of lidocaine at different temperatures on firing rate of the ventral buccal nerve

One-way analysis followed by the Bonferroni test showed a significant difference in the spontaneous activity of neurons $\left(\mathrm{F}_{3,40}=11.01, \mathrm{P}=0.001\right)$ following treatment with lidocaine at different temperatures. As shown in Figure 4A, the neurons' baseline activity did not significantly differ between the BT-Lid. and the RT-Lid. groups. The results revealed that following lidocaine injections at different temperatures, the number of spikes per bin decreased significantly in both the BT-Lid. and RT-Lid. groups compared to their baseline activities $(\mathrm{P}<0.05$, and $\mathrm{P}<0.001$, respectively). Although there was no difference between lidocaine treatments on the number of spikes compared to their baselines, lidocaine at room and body temperature had an inhibitory effect (about $50.3 \%$ and $64.9 \%$, respectively) on the number of spikes. 


\section{Formalin induced hind paw test}
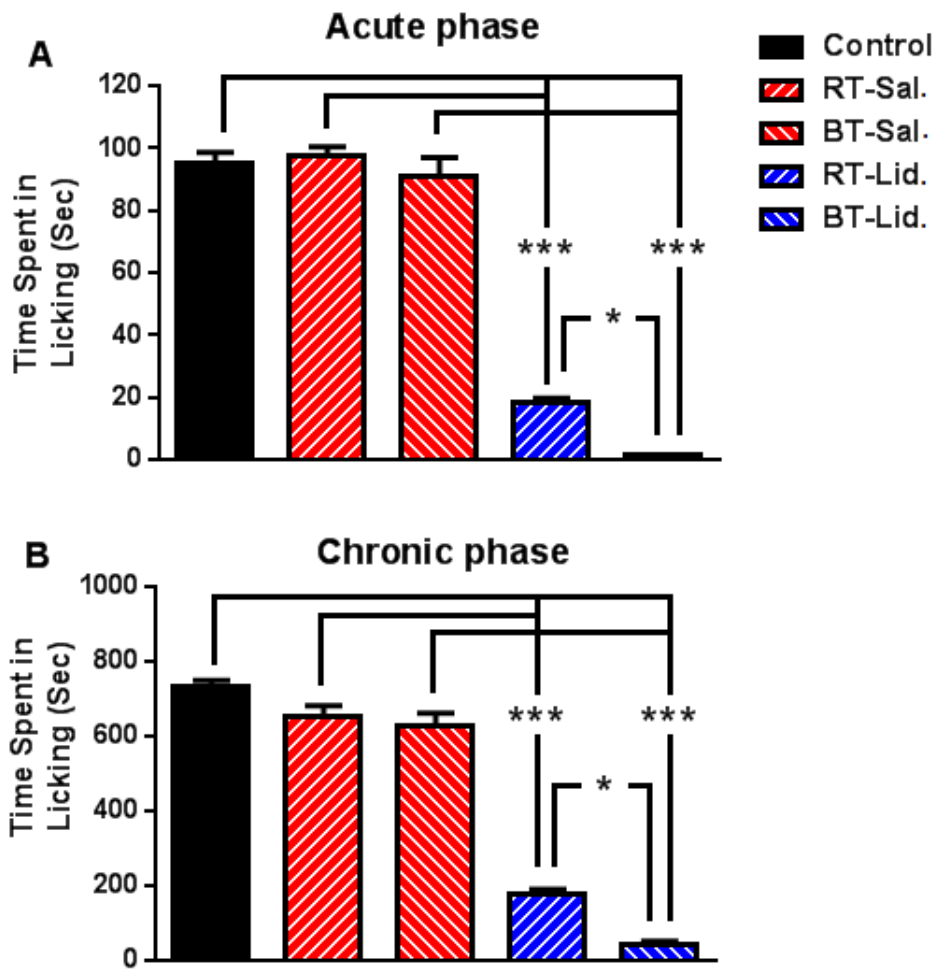

Figure 1. Effect of lidocaine injection at room temperature (RT) and body temperature (BT) on the total time spent licking (s) of the hind paw in the acute (A) and chronic (B) formalin test

The total time spent licking was significantly lower in the BT-Lid. and RT-Lid. groups than in the RT-Sal, BT-Sal, and control groups.

* Significant level for the BT and the RT groups compared to the control groups. Values are Mean \pm SEM $\left({ }^{*} \mathrm{P}<0.05\right.$ and $\left.{ }^{* * *} \mathrm{P}<0.001\right)$.

RT: room temperature; BT: body temperature; Lid.: lidocaine: Sal.: saline.

Further analysis by two-way ANOVA followed by Bonferroni test on the number of spikes per bin indicate that the main effect for treatment $\left(\mathrm{F}_{1,247}=3.89, \mathrm{P}=0.04\right)$ and time $\left(\mathrm{F}_{12,247}=4.3, \mathrm{P}=0.001\right)$ were revealed but not for the interaction of treatment $*$ time $\left(\mathrm{F}_{12,247}=0.037, \mathrm{P}=0.99\right)$. The results indicate that the number of spikes per bin decreased significantly in the BT-Lid. group compared to the baseline at $0-3000 \mathrm{~s}$ after lidocaine injection $(\mathrm{P}<0.01)$, while a significant reduction in the number of spikes per bin was observed at 0-1000, 1250-2250, and $2750-3000 \mathrm{~s}$ in the RT-Lid. group compared to its baseline $(\mathrm{P}<0.05)$ (Figure 4B)

\section{Discussion}

In the present study, the anesthesia success rate was investigated at different temperatures of lidocaine administration. Behavioral and electrophysiological data showed that lidocaine acts better at BT than at RT in reducing formalin-induced pain and the neural activity of the marginal nerve. In formalin-induced hind paw pain, rats were placed in a box with a floor temperature of $37^{\circ} \mathrm{C}$ for $10 \mathrm{~min}$, and they were treated with lidocaine at BT. Therefore, heat could decrease the pain by the gate theory mechanism in the spinal cord (Ropero Peláez \& Taniguchi, 2016). The pain threshold increases as the temperature rises, and also heat can affect metabolism, blood circulation, and muscle spasms (Frölich et al., 2016). In line with the present study, Lundbom et al. showed that after subcutaneous injection of lidocaine into the abdominal cavity of 36 participants, of 9 patients preferred injection at refrigerated temperature, 9 patients endorsed lidocaine at ambient temperature, and the others had less pain with heated lidocaine to $380 \mathrm{o}$ (Lundbom et al., 2017). Moreover, in a meta-analysis in 2011, Hogan et al. reviewed 29 papers and found that warming local anesthetics can reduce pain in intradermal and subcutaneous injections. However, they concluded that the efficacy of warming anesthetic solutions for intraoral injections was not significant (Hogan et al., 2011). The 


\section{Formalin induced orofacial pain}
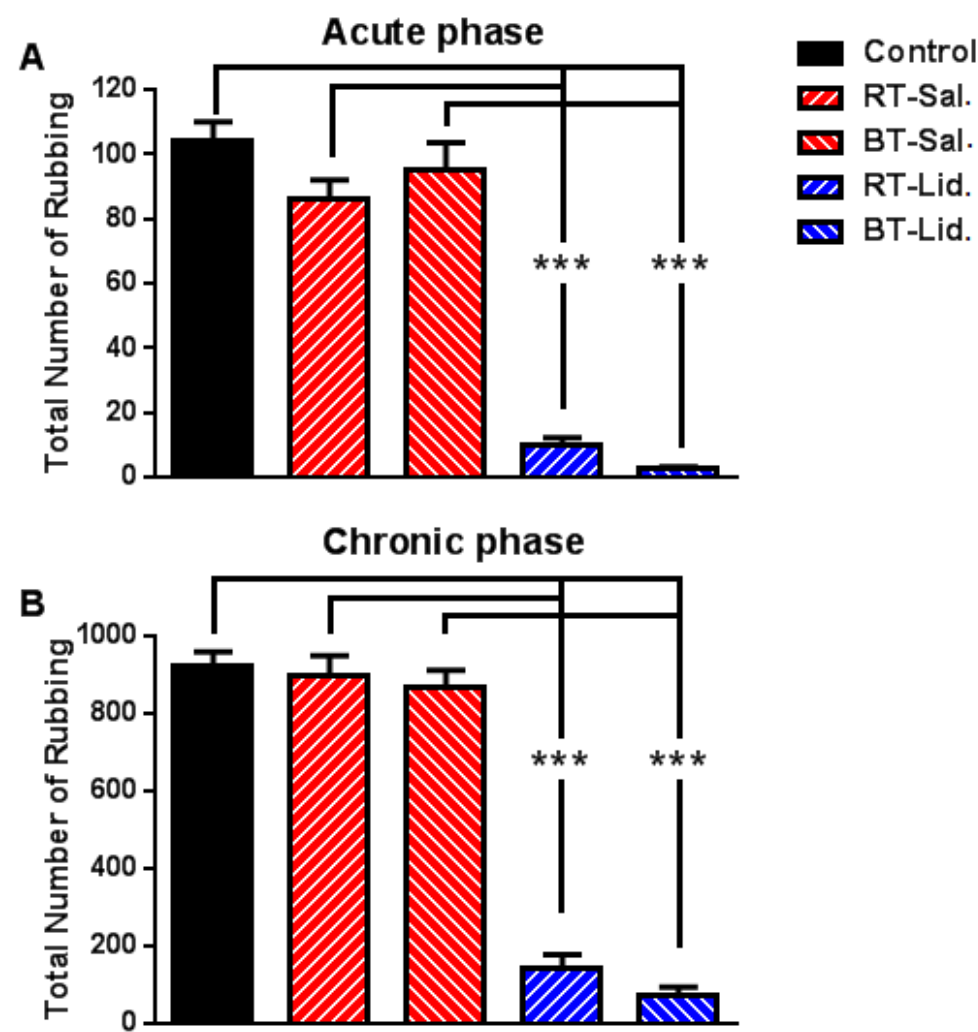

NEUR SCIENCE

Figure 2. Effect of lidocaine injection at room temperature (RT) and body temperature (BT) on the total number of rubbings of the lip in acute (A) and chronic (B) phases of formalin-induced orofacial pain

The number of rubbings was significantly lower in the BT-Lid. and RT-Lid. groups than in the related RT-Sal and BT-Sal and control groups. *a significant level for the BT and the RT groups compared to the control groups. Values are shown as Mean \pm SEM (*P<0.05 and *** $\mathrm{P}<0.001)$.

RT: room temperature; BT: body temperature; Lid.: lidocaine: Sal: saline.

present study showed lidocaine is more effective at BT than at RT in the acute and chronic phases of formalininduced orofacial pain. Previous studies showed that the acute phase is related to the activation of peripheral nociceptors, while the chronic phase of the formalin test involves the continuous activity of primary afferents and increases the sensitivity of dorsal horn neurons (Murray, Porreca, \& Cowan, 1988; Taylor, Peterson, \& Basbaum, 1995). Moreover, intrathecal lidocaine pretreatment before formalin injection in rats decreases c-Fos expression on the dorsal horn of the spinal cord (Inan et al., 2009).

In rodents and humans, trigeminal and facial nerve branches are mixed with cranial nerves that carry sensory information from teeth to the brain (Cobo et al., 2017; Dörfl, 1985; Hwang et al., 2015; König et al., 2007). Previous studies have illustrated that the wide range of neurons from the trigeminal nerve nuclei increases their activity in response to peripheral stimuli, including those from rats' dental pulp (Hamba, Hisamitsu, \& Muro, 1992; Roch, Messlinger, Kulchitsky, Tichonovich, Azev, \& Koulchitsky). The root of the incisor in rats has a wide-open shape; therefore, a dental pulp electrical stimulation may spread out beyond the tooth pulp (Myslinski \& Matthews, 1987). However, ferret canine teeth are more suitable for electrical or thermal stimulation (Ahn et al., 2012). The marginal nerve is more accessible than the cranial trigeminal subnucleus caudalis in rats; therefore, the marginal nerve was exposed to record in vivo neural activity in the present study.

According to the electrophysiology data of the present study, the use of anesthesia at $22^{\circ} \mathrm{C}$ and $37^{\circ} \mathrm{C}$ significantly reduced neuron spikes compared to baselines after the injection of $2 \%$ lidocaine and the warmer lidocaine was more effective than the others. As mentioned, lidocaine 


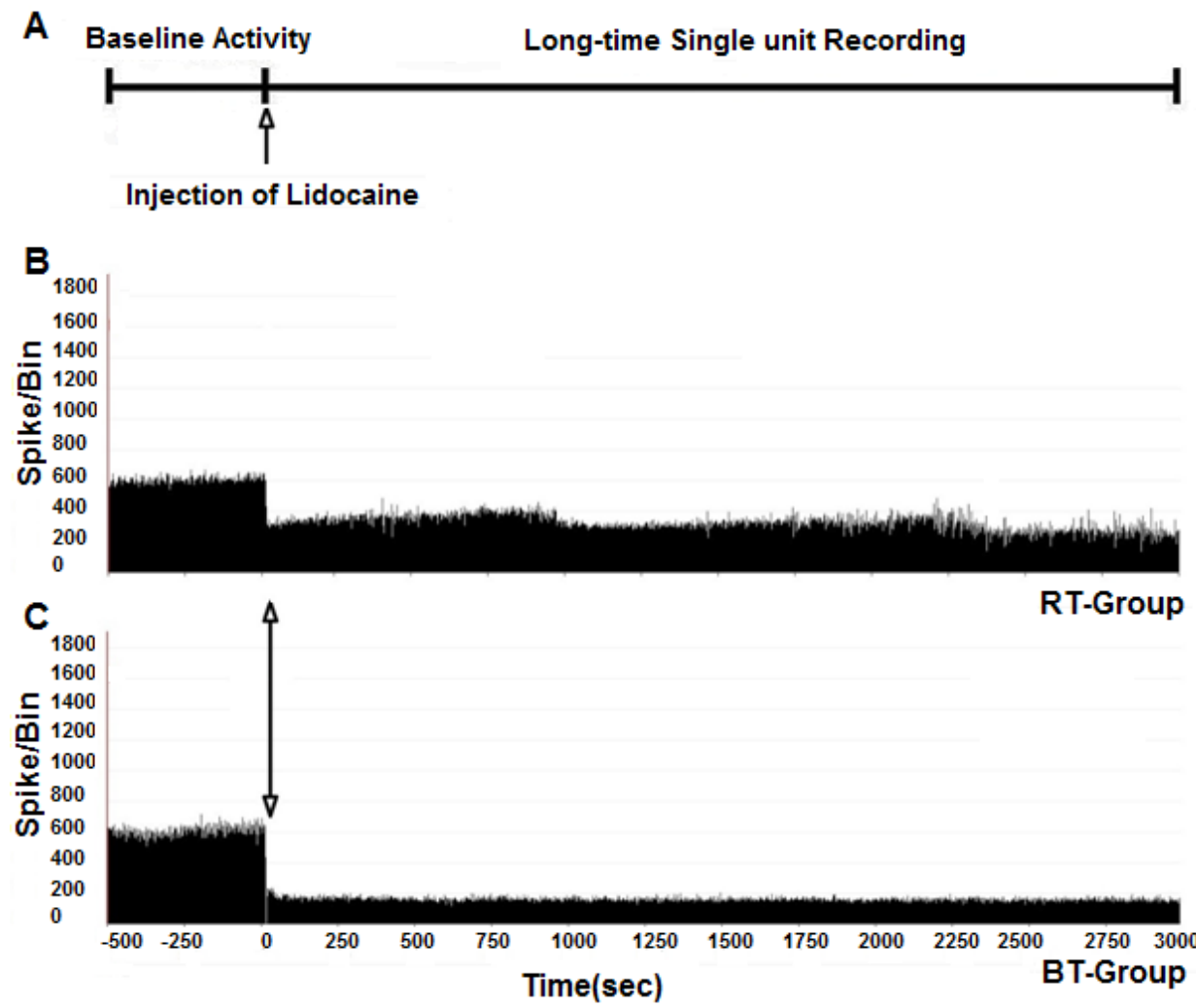

NEUR SCIENCE

Figure 3. Schematic view of the electrophysiology procedure

(A) experiment, (B), and (C) show peri-stimulus time histograms (bin=1 ms) responses of a sample neuron in the room temperature (RT) and body temperature (BT) groups before and after lidocaine injection.

solubility increases with rising temperature (Powell, 1987), and this can be one of the reasons why the solution acts faster when the temperature rises and leads to faster induction of anesthesia. The function of local anesthetics is to block changes in the diffusion of the nerve impulses along the nociceptor by inhibiting sodium channels (Hwang et al., 2015). Moreover, warming the anesthetic agent to body temperature may assist the molecule in infiltrating the nociceptor, consequently contributing to faster blocking of the sodium channels, thereby preventing pain signals by increasing the fluidity of the lipid membrane. Also, lidocaine could cross the nerve membrane more comfortably, achieving effective concentration to produce analgesia more instantaneously (Caterina, Schumacher, Tominaga, Rosen, Levine, \& Julius, 1997).

Finally, successful anesthesia has been described as the lack of response of a volunteer who has received anesthesia to coldness or two consecutive maximum output electric pulp sensibility tests (Sampaio, Carnaval, T. G., Lanfredi, Horliana, Rocha, \& Tortamano, 2012). In dental medicine, the fear of pain attributed to anesthetic injection has been proven to be an obstacle to provid- ing appropriate dental care (Armfield \& Milgrom, 2011; Milgrom, Coldwell, Getz, Weinstein, \& Ramsay, 1997). Although pain cannot be eliminated at the time of injection, it can be significantly relieved. It has been shown that less pain during the procedure accompanies a higher success rate of injection (Hargreaves \& Keiser, 2002). According to the present data, increasing the temperature of lidocaine may optimize the success rate of anesthesia in dentistry. Similar to our report, some studies suggest that warming the anesthetic can reduce injection pain and increase anesthesia success. Pre-warming and buffering the anesthetic solution reduces pain during administration and procedures in maxillary and mandibular teeth (Kurien, Goswami, \& Singh, 2018). Rogers et al. found that using anesthesia at $37^{\circ} \mathrm{C}$ was more comfortable than at room temperature (Rogers, Fielding, \& Markiewicz, 1989). In contrast, other studies have suggested that warming the anesthetic does not affect pain. By studying 44 children, Ram, Hermida, \& Peretz (2002) concluded that there was no significant difference between the pain experienced during the injection in the group injected with a cartridge at room temperature with 
A
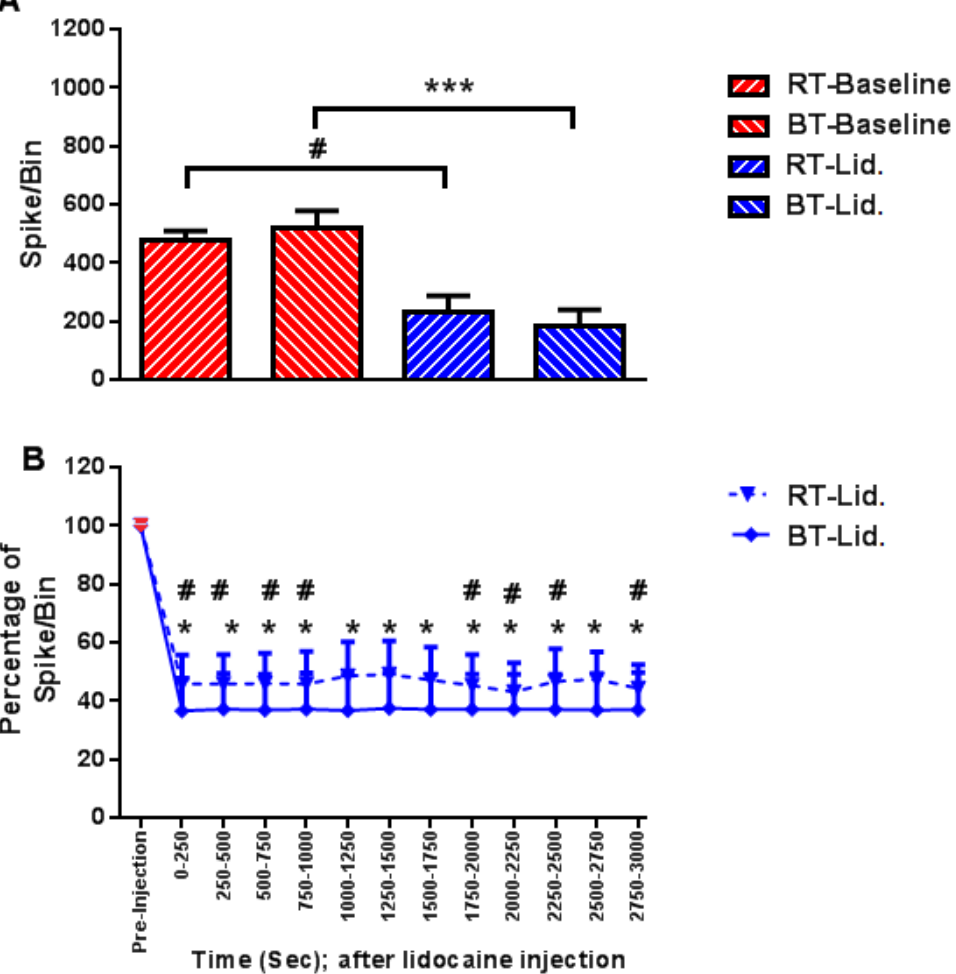

-r. RT-Lid.

$\rightarrow$ BT-Lid.

NEUR SCIENCE

Figure 4. Effect of lidocaine injection at Room Temperature (RT) and Body Temperature (BT) on the total number of spikes (A) and percentage of the number of spikes per bin (B) following single unit recording from the marginal nerve per bin of the marginal nerve

The number of spikes per bin was significantly lower in the BT-Lid. and RT-Lid. groups than their baselines. * and \# significant levels for the BT and the RT groups compared to their related baselines. Values are shown as mean $\pm \mathrm{SEM}\left({ }^{*} \mathrm{P}<0.01\right.$ and $\left.\# \mathrm{P}<0.05\right)$.

a group that received the injection from a warmed cartridge (Ram et al., 2002).

A limitation of this study was that we selected the percentage of formalin based on previous studies (Dubuisson \& Dennis, 1977; Inan et al., 2009; Patel et al., 2013). As there is a correlation between formalin concentration and the rubbing activity (Clavelou, Dallel, Orliaguet, Woda, \& Raboisson, 1995), it is possible that the effects of temperature changes could have been more prominent if the percentage of formalin had been lower. In the electrophysiology phase of this study, only the effect of temperature differences in the absence of a pain stimulus was examined. These issues can be further explored in complementary studies in the future.

\section{Conclusion}

The present study showed that warming the lidocaine cartridges to $37^{\circ} \mathrm{C}$ increased anesthesia success compared to anesthesia-induced at room temperature. Accordingly, a warmed anesthetic cartridge could be used to control pain by increasing the success rate during dental injection and may also apply to clinical studies on humans. Moreover, the models designed in the present study can be used in other studies to compare with other anesthetic solutions or to test the anesthetic effect of materials.

\section{Ethical Considerations}

\section{Compliance with ethical guidelines}

The the Ethics Committee Research Council of Kerman University of Medical Sciences approved this study (IR.KMU.REC.1398.679).

\section{Funding}

The paper was extracted from the Ph.D. dissertation of the first author, Department of Oral and Dental Diseases Research Center, Dental School, Kerman University of Medical Sciences, Kerman, Iran. 


\section{Authors' contributions}

Conceptualization and Supervision: Masoud Parirokh and Mohammadreza Afarinesh; Methodology: Vahid Sheibani and Mohammadreza Afarinesh; Investigation, Writing-original draft, and Writing-review \& editing: All authors; Data collection: Sina Kakooei, Reza Nikzad, Mahshid Mostafavi, And Amir Nekouei; Data analysis: Mohammadreza Afarinesh and Mansoureh Sabzalizadeh.

\section{Conflict of interest}

The authors declared no conflict of interest.

\section{Acknowledgments}

The authors would like to thank the staff of Kerman Neuroscience Research Center in conducting the study.

\section{References}

Adams, E. D., \& Bianchi, A. L. (2008). A practical approach to labor support. Journal of Obstetric, Gynecologic, and Neonatal Nursing, 37(1), 106-115. [DOI:10.1111/j.15526909.2007.00213.x] [PMID]

Ahn, D. K., Doutova, E. A., McNaughton, K., Light, A. R., Närhi, M., \& Maixner, W. (2012). Functional properties of tooth pulp neurons responding to thermal stimulation. Journal of Dental Research, 91(4), 401-406. [DOI:10.1177/0022034511435703] [PMID] [PMCID]

Arendt, K. W., \& Tessmer-Tuck, J. A. (2013). Nonpharmacologic labor analgesia. Clinics in Perinatology, 40(3), 351-371. [DOI:10.1016/j.clp.2013.05.007] [PMID]

Armfield, J. M., \& Milgrom, P. (2011). A clinician guide to patients afraid of dental injections and numbness. SAAD Digest, 27, 33-39. [PMID]

Carstens, E., Kuenzler, N., \& Handwerker, H. O. (1998). Activation of neurons in rat trigeminal subnucleus caudalis by different irritant chemicals applied to oral or ocular mucosa. Journal of Neurophysiology, 80(2), 465-492. [DOI:10.1152/ jn.1998.80.2.465] [PMID]

Caterina, M. J., Schumacher, M. A., Tominaga, M., Rosen, T. A., Levine, J. D., \& Julius, D. (1997). The capsaicin receptor: a heat-activated ion channel in the pain pathway. Nature, 389(6653), 816-824. [DOI:10.1038/39807] [PMID]

Cervero F. (2009). Visceral versus somatic pain: Similarities and differences. Digestive Diseases, 27 (S1), 3-10. [DOI:10.1159/000268115] [PMID]

Clavelou, P., Dallel, R., Orliaguet, T., Woda, A., \& Raboisson, P. (1995). The orofacial formalin test in rats: effects of different formalin concentrations. Pain, 62(3), 295-301. [DOI:10.1016/0304-3959(94)00273-H] [PMID]
Cobo, J. L., Solé-Magdalena, A., Menéndez, I., de Vicente, J. C. \& Vega, J. (2017). Connections between the facial and trigeminal nerves: Anatomical basis for facial muscle proprioception JPRAS Open, 12, 9-18. [DOI:10.1016/j.jpra.2017.01.005]

Courtney, D. J., Agrawal, S., \& Revington, P. J. (1999). Local anaesthesia: To warm or alter the $\mathrm{pH}$ ? A survey of current practice. Journal of the Royal College of Surgeons of Edinburgh 44(3), 167-171. [PMID]

Dörfl J. (1985). The innervation of the mystacial region of the white mouse: A topographical study. Journal of Anatomy, 142, 173-184. [PMID]

Dubuisson, D., \& Dennis, S. G. (1977). The formalin test: a quantitative study of the analgesic effects of morphine, meperidine, and brain stem stimulation in rats and cats. Pain, 4(2), 161-174. [DOI:10.1016/0304-3959(77)90130-0] [PMID]

Frölich, M. A., Banks, C., Warren, W., Robbins, M., \& Ness, T. (2016). The association between progesterone, estradiol, and oxytocin and heat pain measures in pregnancy: An observational cohort study. Anesthesia and Analgesia, 123(2), 396401. [DOI:10.1213/ ANE.0000000000001259] [PMID]

Ganji, Z., Shirvani, M. A., Rezaei-Abhari, F., \& Danesh, M. (2013). The effect of intermittent local heat and cold on labor pain and child birth outcome. Iranian Journal of Nursing and Midwifery Research, 18(4), 298-303. [PMID] [PMCID]

Hamba, M., Hisamitsu, H., \& Muro, M. (1992). Wind-up of tooth pulp-evoked responses and its suppression in rat trigeminal caudal neurons. Brain Research Bulletin, 29(6), 883-889. [DOI:10.1016/0361-9230(92)90160-Y]

Hargreaves, K. M., \& Keiser, K. (2002). Local anesthetic failure in endodontics: Mechanisms and management. Endodontic Topics, 1(1), 26-39. [DOI:10.1034/j.1601-1546.2002.10103.x]

Heaton, J. T., Sheu, S. H., Hohman, M. H., Knox, C. J., Weinberg, J. S., \& Kleiss, I. J., et al. (2014). Rat whisker movement after facial nerve lesion: Evidence for autonomic contraction of skeletal muscle. Neuroscience, 265, 9-20. [DOI:10.1016/j. neuroscience.2014.01.038] [PMID] [PMCID]

Herfst, L. J., \& Brecht, M. (2008). Whisker movements evoked by stimulation of single motor neurons in the facial nucleus of the rat. Journal of Neurophysiology, 99(6), 2821-2832. [DOI:10.1152/jn.01014.2007] [PMID]

Hogan, M. E., vanderVaart, S., Perampaladas, K., Machado, M., Einarson, T. R., \& Taddio, A. (2011). Systematic review and meta-analysis of the effect of warming local anesthetics on injection pain. Annals of Emergency Medicine, 58(1), 86-98.e1. [DOI:10.1016/j.annemergmed.2010.12.001] [PMID]

Hohman, M. H., Kleiss, I. J., Knox, C. J., Weinberg, J. S., Heaton, J. T., \& Hadlock, T. A. (2014). Functional recovery after facial nerve cable grafting in a rodent model. JAMA Facial Plastic Surgery, 16(1), 20-24. [DOI:10.1001/jamafacial.2013.1431] [PMID] [PMCID]

Hwang, K., Yang, S. C., \& Song, J. S. (2015). Communications between the trigeminal nerve and the facial nerve in the face: A systematic review. Journal of Craniofacial Surgery, 26(5), 16431646. [DOI:10.1097/SCS.0000000000001810] [PMID]

Inan, S., Dun, N. J., \& Cowan, A. (2009). Inhibitory effect of lidocaine on pain and itch using formalin-induced nociception 
and 5'-guanidinonaltrindole-induced scratching models in mice: Behavioral and neuroanatomical evidence. European Journal of Pharmacology, 616(1-3), 141-146. [DOI:10.1016/j. ejphar.2009.06.026] [PMID] [PMCID]

Klein, A., Carstens, M. I., \& Carstens, E. (2011). Facial injections of pruritogens or algogens elicit distinct behavior responses in rats and excite overlapping populations of primary sensory and trigeminal subnucleus caudalis neurons. Journal of $\mathrm{Neu}$ rophysiology, 106(3), 1078-1088. [DOI:10.1152/jn.00302.2011] [PMID] [PMCID]

König, H. E., Hans-Georg, H. G., \& Bragulla, H. (2007). Veterinary anatomy of domestic mammals: Textbook and colour atlas. Germany: Schattauer Verlag. https:// books.google.com/ books/about/Veterinary_Anatomy_of_Domestic_Mammals.html?id=QoXiBjSp368C

Kurien, R. S., Goswami, M., \& Singh, S. (2018). Comparative evaluation of anesthetic efficacy of warm, buffered and conventional $2 \%$ lignocaine for the success of inferior alveolar nerve block (IANB) in mandibular primary molars: A randomized controlled clinical trial. Journal of Dental Research, Dental Clinics, Dental Prospects, 12(2), 102-109. [DOI:10.15171/ joddd.2018.016] [PMID] [PMCID]

Lazarov, N. E. (2002). Comparative analysis of the chemical neuroanatomy of the mammalian trigeminal ganglion and mesencephalic trigeminal nucleus. Progress in Neurobiology, 66(1), 19-59. [DOI:10.1016/S0301-0082(01)00021-1]

Luccarini, P., Childeric, A., Gaydier, A. M., Voisin, D., \& Dallel, R. (2006). The orofacial formalin test in the mouse: A behavioral model for studying physiology and modulation of trigeminal nociception. The Journal of Pain, 7(12), 908-914. [DOI:10.1016/j.jpain.2006.04.010] [PMID]

Lundbom, J. S., Tangen, L. F., Wågø, K. J., Skarsvåg, T. I., Ballo, S., Hjelseng, T., et al. (2017). The influence of Lidocaine temperature on pain during subcutaneous injection. Journal of Plastic Surgery and Hand Surgery, 51(2), 118-121. [DOI:10.1080 /2000656X.2016.1194281] [PMID]

McGowan Jr, F. X., \& Davis, P. J. (2008). Anesthetic-related neurotoxicity in the developing infant: Of mice, rats, monkeys and, possibly, humans. Anesthesia and Analgesia, 106(6), 1599602. [DOI:10.1213/ane.0b013e31817330cf] [PMID]

Milgrom, P., Coldwell, S. E., Getz, T., Weinstein, P., \& Ramsay, D. S. (1997). Four dimensions of fear of dental injections. The Journal of the American Dental Association, 128(6), 756-762. [DOI:10.14219/jada.archive.1997.0301] [PMID]

Murray, C. W., Porreca, F., \& Cowan, A. (1988). Methodological refinements to the mouse paw formalin test: An animal model of tonic pain. Journal of Pharmacological Methods, 20(2), 175-186. [DOI:10.1016/0160-5402(88)90078-2]

Myslinski, N., \& Matthews, B. (1987). Intrapulpal nerve stimulation in the rat. Journal of Neuroscience Methods, 22(1), 73-78. [DOI:10.1016/0165-0270(87)90092-6]

Pahlavan, Y., Sepehri, G., Sheibani, V., Afarinesh Khaki, M., Gojazadeh, M., Pahlavan, B., et al. (2013). Study the antinociceptive effect of intracerebroventricular injection of aqueous extract of Origanum vulgare leaves in rat: Possible involvement of opioid system. Iranian Journal of Basic Medical Sciences, 16(10), 1109-1113. [PMCID]
Patel, R. B., Pawar, V. D., Prajapati, K. D., Sonara, B. M., Deshpande, S. S., Shah, G. B., et al. (2013). Anti-nociceptive and anti-allodynic activity of aliskiren in various pain models. $\mathrm{Eu}$ ropean Journal of Pharmacology, 708(1-3), 80-87. [DOI:10.1016/j. ejphar.2013.03.036] [PMID]

Powell, M. F. (1987). Stability of lidocaine in aqueous solution: Effect of temperature, $\mathrm{pH}$, buffer, and metal ions on amide hydrolysis. Pharmaceutical Research, 4(1), 42-45. [DOI:10.1023/A:1016477810629] [PMID]

Ram, D., Hermida, L. B., \& Peretz, B. (2002). A comparison of warmed and room-temperature anesthetic for local anesthesia in children. Pediatric Dentistry, 24(4), 333-336. [PMID]

Roch, M., Messlinger, K., Kulchitsky, V., Tichonovich, O., Azev, O., \& Koulchitsky, S. (2007). Ongoing activity in trigeminal wide-dynamic range neurons is driven from the periphery. Neuroscience, 150(3), 681-691. [DOI:10.1016/j.neuroscience.2007.09.032] [PMID]

Rogers, K., Fielding, A., \& Markiewicz, S. (1989). The effect of warming local anesthetic solutions prior to injection. General Dentistry, 37(6), 496-499. [PMCID]

Ropero Peláez, F. J., \& Taniguchi, S. (2016). The gate theory of pain revisited: Modeling different pain conditions with a parsimonious neurocomputational model. Neural Plasticity, 2016, 4131395. [DOI:10.1155/2016/4131395] [PMID] [PMCID]

Rosivack, R. G., Koenigsberg, S. R., \& Maxwell, K. C. (1990). An analysis of the effectiveness of two topical anesthetics. Anesthesia Progress, 37(6), 290-292. [PMCID]

Sampaio, R. M., Carnaval, T. G., Lanfredi, C. B., Horliana, A. C. R. T., Rocha, R. G., \& Tortamano, I. P. (2012). Comparison of the anesthetic efficacy between bupivacaine and lidocaine in patients with irreversible pulpitis of mandibular molar. Journal of Endodontics, 38(5), 594-597. [DOI:10.1016/j. joen.2012.01.008] [PMID]

Taylor, B. K., Peterson, M. A., \& Basbaum, A. I. (1995). Persistent cardiovascular and behavioral nociceptive responses to subcutaneous formalin require peripheral nerve input. Journal of Neuroscience, 15(11), 7575-7584. [DOI:10.1523/JNEUROSCI.15-11-07575.1995] [PMID] [PMCID]

Vickers, E., \& Punnia-Moorthy, A. (1992). A clinical evaluation of three topical anaesthetic agents. Australian Dental Journal, 37(4), 266-270. [DOI:10.1111/j.1834-7819.1992.tb04742.x]

Yazdkhasti, M., \& Pirak, A. (2016). The effect of aromatherapy with lavender essence on severity of labor pain and duration of labor in primiparous women. Complementary therapies in Clinical Practice, 25, 81-86. [DOI:10.1016/j.ctcp.2016.08.008] [PMID] 